\title{
Dynamic and Tangible Representations in Mathematics Education
}

Published in Laborde C., Laborde J.-M. (2014) Dynamic and Tangible Representations in Mathematics Education In: Transformation - A fundamental Idea of Mathematics Education: A New Approach, Sebastian Rezat; Mathias Hattermann; Andrea Peter-Koop (Eds.), (pp. 187-202) Springer Verlag

\author{
Colette Laborde, Jean-Marie Laborde \\ Université Joseph Fourier, Grenoble, France, Cabrilog, Grenoble, France
}

\begin{abstract}
Dynamic geometry environments offer a new kind of representation of mathematical objects that are variable and behave "mathematically" when one of the elements of the construction is dragged. The chapter addresses three dimensions about the transformations brought by this new kind of representation in mathematics and mathematics education: an epistemological dimension, a cognitive dimension and a didactic dimension.
\end{abstract}

As so often stated since the time of ancient Greece, the nature of mathematical objects is by essence abstract. Mathematical objects are only indirectly accessible through representations (D'Amore 2003, pp. 39-43) and this contributes to the paradoxical character of mathematical knowledge: "The only way of gaining access to them is using signs, words or symbols, expressions or drawings. But at the same time, mathematical objects must not be confused with the used semiotic representations" (Duval 2000, p. 60). Other researchers have stressed the importance of these semiotic systems under various names. Duval calls them registers. Bosch and Chevallard (1999) introduce the distinction between ostensive and non ostensive objects and argue that mathematicians have always considered their work as dealing with non-ostensive objects and that the treatment of ostensive objects (expressions, diagrams, formulas, graphical representations) plays just an auxiliary role for them. Moreno Armella (1999) claims that every cognitive activity is an action mediated by material or symbolic tools.

Through digital technologies, new representational systems were introduced with increased capabilities in manipulation and processing. The dragging facility in dynamic geometry environments (DGE) illustrates very well the transformation technology can bring in the kind of representations offered for mathematical activity and consequently for the meaning of mathematical objects. A diagram in a DGE is no longer a static diagram, representing an instance of a geometrical 
object, but a class of drawings, representing invariant relationships among variable elements: The dynamic parallelogram $A B C D$, constructed on variable points $A, B$, and $C$ represents two relationships of parallelism between two opposite sides, $A B$ and $C D$, on the one hand, $A D$ and $B C$, on the other. It is because the relationships of parallelism are invariant in the dragging, while points and sides vary, that they constitute the mathematical essence of this figure. The dynamic representation expresses, in this example, the generality of the parallelogram. The distinction between drawing and figure is clearly illuminated by DGE and a discussion (initiated by Parzysz in 1988 independently of DGE) took place at the international level, about the complexity of relationships between diagram and figure, in particular in a workshop organized by Straesser in August 1990 at IDM in Bielefeld (Germany) (Strässer 1991, Laborde 1991). A more recent synthesis is presented in Kadunz \& Straesser (2007, pp. 39-46).

The role of representations in the use of digital technologies is essential. Hoyles and Noss (2003) consider digital technologies as "dynamic manipulable and interactive representational forms" that "mediate and are mediated by mathematical thinking and expression" (p. 326). As they stress it, the systems we use to present or represent our thoughts to ourselves and to others, to create and communicate records across space and time, and to support reasoning and computation, constitute an essential part of our cultural infrastructure.

The paper addresses three dimensions about the transformations brought by this new kind of representations:

- An epistemological dimension: the problems faced by software designers when working on the features of direct manipulation of representations of variable mathematical objects

- A cognitive dimension: the way students learn mathematics using this new kind of representation offers a window on their conceptualizations;

- A didactic dimension: how transforming the tasks by taking into account the features of these dynamic representations, in particular of the drag mode, may impact students' learning.

\section{Designing the features of direct manipulation: the case of Cabri 3D}

Direct manipulation has proven to be a key feature to facilitate creative user interaction with a computer and has slowly generalized to most of computer platforms. For educational software nevertheless direct manipulation cannot be designed by chance and has to follow some additional principles. One of them is called epistemic fidelity: the representations of mathematical objects have to avoid any contradiction with the abstract object they are supposed to represent; and this has to be true at the graphical level as at the level of their behavior under direct 
manipulation. Let us elaborate on the difference between interactivity and direct manipulation.

When interacting with a modern computer, the interface is essentially interactive in the sense that the user is "asking" the software to perform something and after the reaction of the computer, s/he asks something again for a next step. The most basic interactivity is offered by so-called "interactive books" giving essentially the possibility to display pages, and by pressing buttons to turn the pages of the books.

This kind of interactivity (unfortunately still widely spread, -especially through Internet-) is easy to develop and leads to a form of "impoverishment" of the user interface, with the generalization of the use of Internet. In contrast authentic direct manipulation software is mainly not driven by the press of buttons, or by the filling of dialogs (or forms) or by typing command lines. It offers an interface where the user is invited to directly act on the mathematical objects. Actually, the action is on the representation of an object or an abstract entity; nevertheless if the implementation is sophisticated enough and if the interaction turns to be of direct engagement type (Schneidermann 1983), eventually the user perceives the representation of the object and the abstract object itself as already noticed by the five main designers of the Star Machine (Smith et al. 1982).

The need for extending the benefit of direct manipulation present in many 2D environments just followed the first introduction of direct manipulation geometry software of Cabri type. Recall that Cabri actually stands for "Cahier de BRouillon Interactif", somehow "Interactive Sketchpad".

The need for extending direct manipulation from 2D to 3D cannot be achieved without a deep transformation of the environment under several aspects: the mathematical problems to be solved for the representing dynamic 3D objects are different and for most of them even still open.

Two kinds of new problems arise: representing 3D objects with a meaningful depth and representing their behaviour in the drag mode; the manipulation of 2D objects with a mouse in a $2 \mathrm{D}$ screen is natural but becomes problematic for $3 \mathrm{D}$ objects: how can the mouse capture the depth of the space?

\subsection{Complexity}

A very common idea about extending a $2 \mathrm{D}$ environment to $3 \mathrm{D}$ is to think that this could be achieved (somehow) merely by adding an additional coordinate to the internal representation of the objects at the level of their data structure: essentially this would be then a trivial task. Actually as it is well known by mathematicians, 3D objects are "essentially" more complex than 2D objects: in most of the cases, augmenting the dimension leads to some complexity increase, even if eventually in higher dimension the situation might show more regularities. In 3D, many "basic problems" are still open. Let us mention the classification of quadrics. In 2D there are only 3 conics: ellipses, parabolas and hyperbolas. In 3D, i.e. for 
quadrics, nobody has yet found any really "elegant" classification that would satisfy everybody. Another example could be the conjecture about the probable existence, for any convex polyhedron, of its unfolding as a net of not overlapping connected (convex) polygons. In 2D "to follow", in a reasonable way, the intersection of 2 conics is not an easy task (actually many of the DGE cloned from the main ones, fail in trying to dynamically follow, in rather simple cases, the intersection points of a circle with a straight line!). In 3D nobody knows (yet and apparently) how to dynamically follow the intersections of a quadric with a line and even less the intersection curves of two quadrics.

\subsection{Representing dynamic 3D objects}

A first new problem arising when moving from 2D to 3D deals with the choice of the perspective.

In classroom the so-called cavalier perspective has been the most popular way for representing 3D scenes. The main reason for that is that it is easy to create perspective drawings using the rules of the cavalier perspective. Recall that this perspective is governed by the fact that it is a parallel (yet not orthogonal) perspective (the observer is at infinity, and parallel lines are still parallel in the perspective drawing). In addition there is a plane in which objects are in real size and actually lines perpendicular to this plane are represented as oblique lines in the drawing. This is really different from the "natural" perspective as introduced by the painters at the time of the Renaissance (e.g. Alberti) and which today can be considered as realized by high quality camera lenses.

Below (Fig. 1) is a cube, in "natural perspective" versus the same cube in cavalier perspective: if we would animate the cube to rotate it round its vertical axis of rotational symmetry, the cube in natural perspective would keep its "cubic" shape, but, in cavalier perspective, the cube would actually somehow pulse as its shape would change during the various phases of the rotation. Cavalier perspective is thus in conflict with the user's perception about the cube as a solid object.

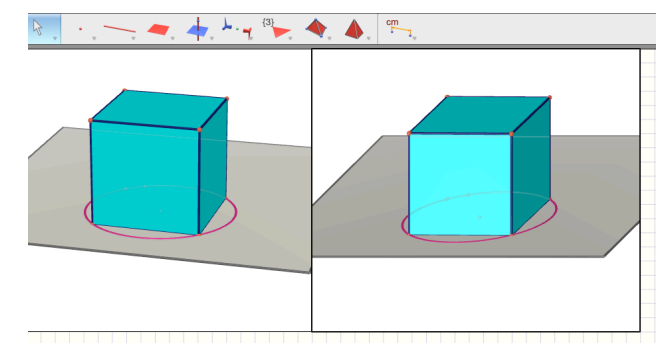

Fig. 1 On the left a cube in natural perspective, on the right the same cube in cavalier perspective. 
Therefore we assumed that natural perspective favours the appropriation of the figure by students and users more generally. This is the reason why in Cabri 3D, the default perspective, is actually a natural perspective. Its characteristics match to the view of an object of approximately $40 \mathrm{~cm}$ in size hold (as in the hand of the user) at a distance of $50 \mathrm{~cm}$. This is in contrast to some other software that by exaggerating the perspective effect (for some artistic purpose?) apparently does not favor the appropriation just mentioned. In the figures above, one can clearly understand how, somehow, cavalier perspective is an attempt to look at an object from two points of view at once: from the front and (here) from the right. Actually this kind of representation has developed in many cultures, ranging from the ancient Egypt to China and Japan.

Let us stress that this choice is not shared by other 3D environments. The latter generally favour either the parallel perspective for easiness of the computations or a strong perspective effect by taking advantage of the facilities of computer graphical cards.

\subsection{Rendering $3 D$ mathematical objects}

There is not much space here to address all specificities of the rendering of 3D mathematical objects (lines, planes, spheres, cylinders...), some of them being "in nature" infinite. Let us just mention the case of the plane, an infinite object. In textbooks, planes are most of the time represented using a rectangle to display a "portion" of the plane. It is also worth to note that textbooks present only a really limited number of figures. Space geometry textbooks display hardly more than 10 different types of 3D situations we could consider as stereotypic. Among them one is the illustration of the famous "théorème du toit", stating that if 2 planes intersect a plane along 2 parallels lines, their intersection is a third parallel line (Fig. 2).

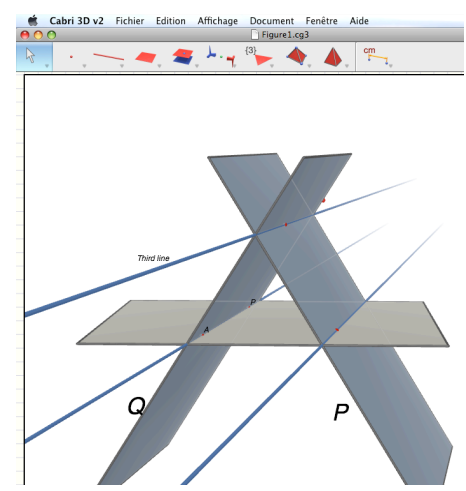

Fig. 2 - Two planes intersecting in a third parallel line 
In textbooks, which display only a static image, one can easily agree about "the good rectangle" taken to represent each plane. When things turn to be dynamic there is no "natural" way to "follow" the plane as it evolves. This is the reason why some $3 \mathrm{D}$ geometry software considering that planes are essentially infinite, do not limit their representations. A plane (up to the special case of being viewed as a line, in French "de bout") covers the whole screen and, in such environments, practically cannot be "seen" and so is not directly represented. We do not consider that this is a good idea for learners. In Cabri after various attempts we decided to represent a plane as a rectangle (in some earlier version as a parallelogram) presenting a certain amount of thickness, in the very same spirit as when Hilbert and Cohn-Vossen designed their 3D figures for their famous "Anschauliche Geometrie" (Hilbert and Cohn Vossen 1952).

\subsection{Manipulating 3D objects}

One of the first things to be considered in order to directly manipulate objects in space is to have a mouse able to drive a point in 3D. Ordinary mice are essentially 2D, though expensive 3D-mice have existed for a while. Because such 3D devices are not expected to be available soon neither in the regular classroom nor at home, we have been looking for various solutions based on an ordinary mouse combined with modifiers (at keyboard level). Actually in Cabri3D the metaphor of the old "typewriter" is used: as long as the shift key is not pressed, the mouse simulates a displacement in some horizontal plane and if the user presses the shift key (implying a vertical movement of the carriage on antique typewriters) the mouse movement is interpreted as a movement along a vertical axis. Note that other environments may have done another choice like Archimedes Geo 3D in which pressing the shift key provokes a move orthogonal to the screen plane. For reasons of making sense for users, Cabri 3D does not permit any arbitrary rotation of the scene (as looking at the scene in some upside down way) and the horizontal reference plane always stays horizontal and consequently verticality is preserved.

All these choices made on the basis of epistemic and ergonomic reasons must then be confronted with the real use by teachers and students. Let us mention here a thorough study (Hattermann 2011) analyzing the use of Archimedes Geo 3D and Cabri 3D by university students, giving evidence of various aspects of the ways of using these environments for solving different kinds of problems. It is worth mentioning that students solved problems more rapidly in Cabri 3D than in Archimedes Geo 3D (p. 164) without a clear evidence of an effect due to a particular feature of the interface of both software environments. It may be the combination of several aspects of the whole interface which played a role. 


\section{A cognitive dimension: dynamic diagrams as a window on students' ideas}

Interacting with dynamic diagrams transforms the usual ways of acting on mathematical objects into new ones. Because students cannot directly apply usual paper and pencil routines in dynamic geometry environments, they have to make decisions about actions. These decisions are influenced by their conceptions about mathematical objects. Therefore placing students in unfamiliar conditions may reveal their own ideas and conceptions about mathematical objects.

Many research works have been carried out on students solving tasks in dynamic geometry environments. In particular, the way in which students drag as they solve geometry problems in DGE was investigated by several researchers. Hölzl (1996) identified the "drag and link approach" in students' construction processes of Cabri diagrams. Students relax one condition to do the construction and then drag to satisfy the last condition. They obtain a diagram visually correct and want to secure the diagram by using the redefinition facility of Cabri. But often it does not work because of hidden dependencies that are not considered by the students. Although Hölzl does not refer to instrumentation, this "drag and link approach" would be called an instrumentation scheme in terms of Vérillon and Rabardel (1995). The students constructed an instrumentation scheme not compatible with the functioning of Cabri.

Arzarello et al. (1998a \& b) identified different kinds of dragging modalities that were not all referring to an organized experimentation: "wandering dragging", "lieu muet" dragging, and dragging to test hypotheses. Wandering is just moving without a predefined aim for searching for regularities whilst "lieu muet" dragging refers to dragging in such a way that some regularity in the drawing is preserved. The dragging to test hypotheses obviously presupposes that regularities have already been detected which are not systematically tested. Goldenberg (1995) notes that often students do not know how to conduct experiments and are unsure what to vary and what to keep fixed. Thus a student's purposeful move from "wandering dragging" to "lieu muet" dragging represents a cognitive shift. Restrepo (2008) who investigated in depth the instrumental genesis of the drag mode by $6^{\text {th }}$ graders over one school year, concluded that the genesis lasts over a long time made of several steps.

From all these investigations, it appears that the power of the drag mode in exploration is not spontaneously mastered by students because the mathematical meaning itself of the drag mode is not yet constructed by students. As early claimed by Strässer (1992), dragging offers a mediation between drawing and figure and can only be used as such at the cost of an explicit introduction and analysis organized by the teacher. Transforming the interaction between student and geometric figures turned out to reveal that the notion of geometric figure as a set of relationships between variable elements is not appropriated by students. It may imply that students do not necessarily recognize the mathematics they learned 
in paper and pencil environment. Mathematics itself may be changed in students' eyes by a dynamic geometry environment.

Below an investigation on the construction of a proof by $9^{\text {th }}$ graders is reported (Abd El All 1996) giving evidence that even a known theorem is transformed in a dynamic geometry environment for students.

\subsection{Students' conception of a theorem}

All students of a class $\left(9^{\text {th }}\right.$ graders) were given the following tasks. They worked in pairs. The work of four pairs was observed and audio-recorded.

\section{Task 1 (Fig. 3)}

Students were given a rectangle $A B C D$ and the quadrilateral $I J K L$ of the midpoints of the sides of $A B C D$ in a paper and pencil environment. They had to determine the nature of $I J K L$ and to justify their answer. All students found that it is a rhombus.

\section{Task 2}

Then they had to predict whether $I J K L$ would remain a rhombus in any movement of $B$ which does not preserve $A B C D$ as a rectangle. All students predicted that $I J K L$ will not be any longer a rhombus.

\section{Task 3}

They were given a rectangle $A B C D$ in Cabri. Then they had to construct the circle with center $D$ and radius $A C$ and to redefine $B$ as belonging to the circle. They were asked. "Is $I J K \mathrm{~L}$ still a rhombus?" (Fig. 4)

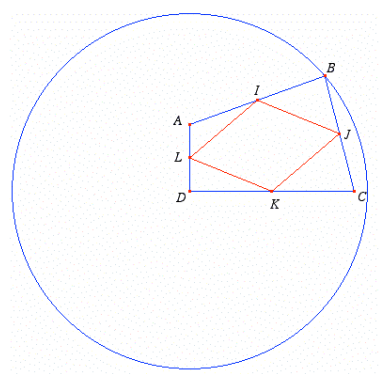

Fig. 3 Rhombus in a rectangle Fig. 4 Rhombus in a quadrilateral with congruent diagonals 
The sequence of questions was designed with the intention to favour the need of having recourse to proof. In a computer environment, the need for proof cannot any longer be favoured by the uncertainty of the result. It may arise for intellectual motives because the student wants to know why a phenomenon takes place. As pointed out by the Piagetian perspective, a means of provoking this intellectual curiosity may be caused by conflict between what the learner believes or predicts and what actually happens. Such a conflict may be achieved by asking the students to predict properties of the diagram before allowing them to check on the computer, as in this problem. In task 1, we expected that students would prove that $I J L K$ is a rhombus by using the specific properties of a rectangle (theorem of Pythagoras, properties of reflection, congruence of right angle triangles) rather than using the more general property of the midpoint segment that is valid even if $A B C D$ is no longer a rectangle. In task 2 we expected them to predict that $I J K L$ is no longer a rhombus as they probably would have justified in task 1 that $I J K \mathrm{~L}$ is a rhombus by using properties of a rectangle. In task 3 they should be very surprised by observing that $I J K L$ remains a rhombus and would be eager to understand why. This is why they were not asked to justify what they observed. We expected that from the strength of the contradiction would arise the need of justifying.

This is exactly what happened. Students were so surprised to discover that $I J K L$ was a rhombus although $A B C D$ was not a rectangle that they became eager to prove why without being asked in an explicit way to do so. However it took time for them to construct a justification. We could observe that the variability of the diagram created several difficulties for students. We comment here on the effect of variability on the use of a theorem. Some students did recognize that $I J$ was the segment joining the midpoints and evoked the property of this segment but they were not sure about the validity of using the theorem when the diagram moved. V. and L. for example evoked the theorem of the midpoint segment but did not dare using it. Pushed by the observer, they selected a triangle and V. looked carefully at the triangle and the midpoint segment when point $B$ was dragged. She expressed her satisfaction:

"The theorem of midpoints moves, yes it moves. It works even if we move"

L. confirmed: " the midpoint theorem it works"

V.: "it works the same way"

$\mathrm{V}$. even tried to justify the invariance of the property in the drag mode:

"they are all the same because there is always the same length. AC it is two times that. It

is always two times that. It is always two times that and it works there all the time even if

we move anyway."

A student of another pair wrote at the end of their proof: "As $D B$ is always the radius, this proof is always right" and then the partner added: "for any position of $B$ "

For these students a proof seems to be carried out only for a particular instance of the diagram. From the work in Cabri the problem of the shift from proving one instance to proving all instances arose for them. According to Netz (1999) Greek proof was rather done on a generic example than in a general case. The validity of the general statement was claimed at the end of the proof in the final part called 
Sumperasma. The expression of the validity claimed by students for all instances obtained by the drag mode can be compared with the expression of the sumperasma in the Greek proof.

In this example, Cabri provided a window (Noss \& Hoyles 1996) on the conceptions of students about proof but the complexity introduced by the variability of the diagram acted as a catalyst for change in this conception for students such as V. and L. who became aware of the fact that a theorem may be valid for a moving diagram since the relations between elements remain unchanged. Questioning the validity of the theorem under the drag mode led the students to focus their attention to the relationships between elements of the figure. They learned from the complexity brought by the computer environment that offered to the students another window on mathematics (Noss \& Hoyles 1996). This point of view was supported by several researchon Computer Algebra Systems (CAS) used as a lever to promote work on the syntax of algebraic expressions (Artigue 2002, p. 265, Lagrange 2002, p. 171, or DGE assisting pupils to distinguish the properties of a rhombus from those of a square, Hoyles \& Jones 1998).

\section{A didactic dimension: transforming the tasks by making use of the features of the software}

\subsection{The a didactical milieu}

The idea of a technological environment that has to be explored and that is interacting with the learner can be linked to the notion of "adidactical milieu" in the theory of didactic situations by Brousseau (1997). In this latter theory, knowledge is constructed by the student as a solution to a problem for which the constructed knowledge item provides an efficient solving strategy. The student does not solve the problem for satisfying the expectations of the teacher but because it is a genuine problem for him, a problem of the same kind as problems encountered in real life outside classroom. The only difference is that real life problems are not organized by a teacher in order to promote learning. But although it is designed by the teacher, a problem in an adidactical situation is experienced by the student as a real life problem. In the core of the notion of adidactical situation is the notion of "adidactical milieu". An "adidactical milieu" offers information and means of action to the student and reacts by providing feedback to his/her actions. It can be of material nature as well as of intellectual nature.

We do not claim that dynamic environments like Cabri II Plus and Cabri 3D provide an "adidactical milieu" but an adidactical milieu can be organized and based on them for at least two main reasons: 
- the available tools allow the user to perform mathematical operations on the representations of the mathematical objects

- the feedback offered by the drag mode allows the user to check whether his/her constructions are done by using mathematical properties and relations and are not simply visually done .

Numerous examples of construction tasks with Cabri I, Cabri II or Cabri II Plus are given in the literature and show how the first solving strategies of students are visual and evolve towards more geometrical constructions through the drag mode playing a double role. The drag mode invalidates purely visual constructions and also provides information about the geometrical behaviour of objects (Hoyles and Noss and Hoyles 1996, p.125; Jones 1998, pp. 79-82). In these construction tasks, geometrical knowledge is efficient since it is the only way to build a construction which is "drag mode proof". As it is possible to configurate the software and to make available a restricted range of default tools or new tools obtained as macroconstructions, the designers of construction tasks can thus promote the use of specific properties by the students and contribute to learning through the organized "adidactical milieu". An eloquent example is given by the task of drawing a perpendicular line to a line without the tool "Perpendicular" but with transformation tools, in particular the "reflection tool".

\subsection{Example of an adidactical milieu in Cabri 3D}

A more recent example (Mithalal 2010) is given here about a construction task in Cabri 3D. Cabri 3D is used to create an adidactical milieu fostering the move by students from a pure global visualization of a solid object, called iconic visualization by Duval (2005), to an analytical breaking down of a solid object into parts interrelated through geometrical relationships called dimensional deconstruction by Duval (2005).

Grade 10 students of two classrooms using Cabri 3D for learning 3D geometry (Mithalal 2010) were faced with the following activity at the beginning of the teaching of 3D geometry and after an introduction to the use of Cabri 3D: A cube with a triangular cross section was given on the screen and the students had to reconstruct the missing vertex so that it remains a vertex even when the cube is enlarged or dragged (Fig. 5). 

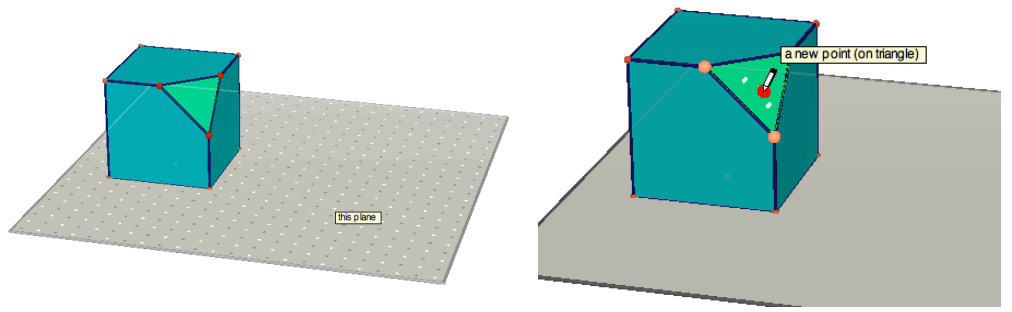

Fig. 5 A truncated cube

Fig. 6 A point visually located

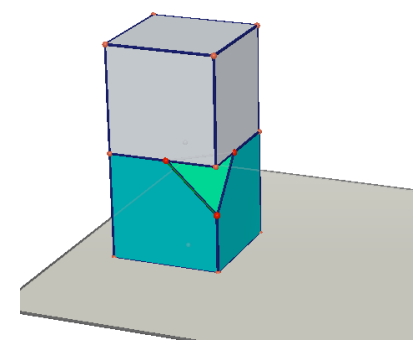

Fig. 7 Adjusting a second cube

In order to foster learning, students were asked to find several solving strategies and tools were withdrawn from Cabri 3D once visual strategies appeared and once it was observed that these strategies did not produce robust vertices against dragging. It was expected that the added constraints would lead students to move to geometric characterizations of the missing vertex.

Producing a vertex by a purely visual strategy is not as easy in Cabri 3D as in paper and pencil environment. If the user attempts to put a point by eye at the desired location, Cabri 3D proposes to create the point on the triangular crosssection (Fig. 6).

Visual strategies must be a little more elaborated and include some geometric components. An example of such a semi-visual strategy is creating a cube on the top of the original cube by visually placing its center at the center of the squared top face and putting one of its vertices to a vertex of the original cube and then visually adjusting this second cube so that its bottom face is coinciding with the top face of the original cube (Fig. 7). Of course a vertex reconstructed in this way is not "dragging resistant". It was decided to withdraw the tool cube from Cabri 3D after such this strategy was proposed by students.

Then strategies conceiving the vertex as intersection of straight lines or of planes were expected in a second phase after semi-visual strategies. Students proposing such strategies were asked to find other strategies without using the tool "point". 
The attentive observation of 30 pairs of students shows that few of them resorted in a first phase to visual or semi visual strategies. Some of those students tried to create a tetrahedron based on the triangular cross section with a fourth vertex providing the missing vertex of the cube. Non iconic visualization clearly underlies such a strategy. Students intended to reconstruct the entire cube as a material entity.

The most prevailing spontaneous strategy was to construct the missing vertex as the intersecting point of the three straight lines supporting the segments adjacent to the cross section (although two lines would be enough). The fact that often three lines and not two were constructed can be interpreted as a strategy inherited from paper and pencil environment mixed with iconic visualization. The three lines allowed students to restore the original representation of the whole cube in paper and pencil environment. Some students then moved to the construction of the vertex as the intersecting point of planes or of a plane and a line. They took advantage of the possibility of using 2D objects in Cabri 3D and extended the intersection strategy. The use of a plane supporting a face moved them away from a purely iconic visualization of the cube and very often when using a plane and a line, the vertex was constructed only with one plane and one line and not with two planes and one line and two lines and one plane. Through the instrumental deconstruction of the cube made possible by Cabri 3D, students moved towards a non iconic visualization.

Finally it must be stressed that after the tool "point" was withdrawn, some students constructed the vertex using geometric transformations like point symmetry or translation: the vertex was constructed as the reflected image of another vertex with respect to the center of a cut face of the cube, or it is the image of a vertex in a translation with the vector which is defined by a side of the cube.

Construction tasks in dynamic geometry environments are thus transformed. They become in a way more demanding since they require the use of geometrical knowledge to be solved and can be more difficult for students. However the drag mode invalidating strategies may encourage the students

\subsection{Tasks specific to dynamic geometry}

Over the twenty years of existence of the Cabri technology, the design of "adidactical milieu" led to experiment with new kinds of tasks that cannot exist in paper and pencil environments, in particular:

- "black box" tasks in which dynamic constructions are given to students who must reconstruct them again so that the reconstructions have the same behavior in the drag mode as the original ones ;

- prediction tasks in which students must predict without dragging what will happen if a specific point is dragged. 
These tasks of a new kind are based on the same idea of taking advantage of the transformation of the nature of diagrams in dynamic geometry environments, namely taking advantage of the variable nature of the diagrams controlled by the mathematical model underlying the software program.

In these tasks students explore and interact with the environment. Through feedback and the available tools they will develop strategies involving geometrical knowledge. The fact that it is the environment and not the teacher who reacts contributes to make the problem analogous to a genuine problem for students. In a black box task, students can experiment on the given construction by adding elements and dragging, in order to find the relationships between its elements. The nature of the mathematical activity of the student is changed and becomes more of an experimental activity in which hypotheses are made and checked by experimenting. In prediction tasks, students must resort to geometrical knowledge to be able to predict the behavior of the construction in the drag mode. Then the predictions can be checked by dragging a point of the construction.

With the extension of Cabri tools to algebraic and graphing tools, the design of tasks making use of the Cabri features went beyond geometry. For example, Falcade (Falcade et al. 2007) designed a milieu for constructing the notion of graph of a function as expressing the covariation of two variables, the first one independent and the second one depending on the first one. Moreno (2006) designed a milieu in which the students had to find the ordinary differential equation of a family of dynamic curves, by exploring the variation and the invariant elements of this family in the drag mode, a kind of black box task in calculus.

\section{Transformations}

In the XIXth century, human knowledge led to the design of tools for the mechanization of human activity. At the end of the XXth century, human knowledge could be embarked in technology modelling domains of theory. In dynamic mathematics environments, human knowledge is embarked in the representations of theoretical objects which behave according to the theoretical model underlying the technology independently of the wishes of the user as soon as the latter has constructed them. One could say that a transformation of a new kind took place. It does not lie only in the creation of artefacts embarking knowledge but also in the creation of artefacts offering a dynamic model of theoretical objects. In terms of the Vygotskian perspective, the creation of artefacts embodying theoretical knowledge at a higher degree than before may affect the nature of the psychological tool constructed by the human. It may extend the role of these psychological tools on the mental activity of the individual. This is very apparent in the reaction of students L. and V. when they discovered that the theorem "moved", i.e. was valid for every occurrence of the 
diagram. The use of a dynamic construction led them to consider a theorem as an invariant statement about variable objects.

The teaching of mathematics can take advantage of the transformation of the offered representations of mathematical objects by changing the kind of tasks given to students for fostering learning as described in section 3. However the role of the teacher is still essential. The students may not be able to solve the new kind of tasks that are more demanding in terms of knowledge and need help from the teacher. Once students have solved the task, the teacher may contribute to an internalization process by organizing social interactions and collective discussions in the classroom, intervening in order to transform the meaning of what has been done on the computer into a meaning that can be related to the "official" mathematical meaning. This process of semiotic mediation based on this new nature of representations was theorized and extensively experimented by Mariotti (2001) (see also Mariotti's chapter in this book).

The industrial revolution affected deeply the human society. The transformations brought by knowledge technology also affected the society. However it influenced school in a minor way. One missing link between this deep transformation of technology and school is certainly pre and in-service teacher education and accompanying measures for taking advantage in everyday teaching of this transformation of representations of mathematical objects.

\section{References}

Abd El All, S. (1996). La géométrie comme un moyen d'explication de phénomènes spatiographiques: une étude de cas, Mémoire de DEA de Didactique des Disciplines Scientifiques, Grenoble: University of Grenoble 1, Laboratoire Leibniz-IMAG (1996)

Artigue, M. (2002). Learning Mathematics in a CAS environment: the genesis of a reflection about instrumentation and the dialectics between technical and conceptual work. International Journal of Computers for Mathematical Learning, 7.3, 245-274

Arzarello, F., Micheletti, C., Olivero, F., Robutti, O. \& Domingo, P (1998a). A model for analysing the transition to formal proofs in geometry. In A. Olivier \& K. Newstead (Eds.), Proceedings from the 22nd Annual Conference of the International Group for the Psychology of Mathematics Education (Vol. 2, pp. 24-31). South Africa: University of Stellenbosch.

Arzarello, F., Micheletti, C., Olivero, F., Robutti, O., Paola, D., \& Gallino, G. (1998b). Dragging in Cabri and modalities of transition from conjectures to proofs in geometry. In A. Olivier \& K. Newstead (Eds.), Proceedings of the 22nd Conference of the International Group for the Psychology of Mathematics Education (Vol. 2, pp. 32 -39). South Africa: University of Stellenbosch.

Bosch, M. \& Chevallard, Y. (1999). La sensibilité de l'activité mathématique aux ostensifs Recherches en didactique des mathématiques, 19.1,77-124.

Brousseau, G. (1997). Theory of Didactical Situations in Mathematics. (N. Balacheff, M. Cooper, R. Sutherland, \& V. Warfield, Trans. and Eds.). Dordrecht: Kluwer.

D'Amore, B. (2003). Le basi filosofische, pedagogiche, epistemologiche e concettuali della Didattica della Matematica, Bologna, Italy: Pitagora Editrice

Duval, R. (2000). Basic issues for research in mathematics education, In T. Nakahara, M. Koyama (Eds.), Procedings of the $24^{\text {th }}$ Conference of the International Group for the Psychology of Mathematics Education, (Vol 1, pp. 55-69) Hiroshima: Hiroshima University 
Duval, R. (2005). Les conditions cognitives de l'apprentissage de la géométrie : développement de la visualisation, différenciation des raisonnements et coordination de leurs fonctionnements, In: Annales de didactique et sciences cognitives, 5-53, Strasbourg, France: IREM, Université Louis Pasteur.

Falcade, R., Laborde, C., Mariotti, A. (2007). Approaching functions: Cabri tools as instruments of semiotic mediation, Educational Studies in Mathematics, 66.3, November 2007, 317-333

Goldenberg, E. P. (1995). Rumination about dynamic imagery. In R. Sutherland \& J. Mason, (Eds.), Exploiting Mental Imagery with computers in mathematics education, (pp.202-224). Heidelberg: Springer Verlag, NATO ASI series F, Vol 138

Hattermann, M. (2011). Explorative Studie zur Hypothesengewinnung von Nutzungsweisen des Zugmodus in dreidimensionalen dynamischen Geometriesoftwaresystemen. Giessen: Dissertation der Justus Liebig Universität

Hilbert, D., Cohn-Vossen, S. (1952). Geometry and the Imagination, New York: Chelsea Publishing Co., 1952, (2nd edition, 1990), translation by P. Nemenyi of Anschauliche Geometrie, Berlin, 1932: Springer-Verlag.

Hölzl, R. (1996). How does the dragging affect the learning of geometry ? International Journal of Computer for Mathematical Learning 1(2) 169-187.

Hoyles, C. \& Jones, K. (1998). Proof in dynamic geometry contexts In: Perspectives on the Teaching of Geometry for the $21^{\text {st }}$ century-An ICMI study, C. Mammana \& V. Villani (eds.) (chap. 4, section II, pp. 121-128) Dordrecht: Kluwer Academic Publisher

Hoyles, C., \& Noss, R. (2003). What can digital technologies take from and bring to research in mathematics education? In J. Bishop, K. Clements, C. Keitel, J. Kilpatrick, \& F. Leung (Eds.), Second International Handbook of Mathematics Education (Part 1; pp.323-349). Dordrecht, The Netherlands: Kluwer Academic.

Jones, K. (1998). Deductive and intuitive approaches to solving geometrical problems. In C. Mammana and V. Villani (Eds.) Perspectives on the Teaching of Geometry for the 21 st century: An ICMI study (pp. 78-83). Dordrecht, The Netherlands: Kluwer Academic Publishers.

Kadunz, G. \& Straesser, R. (2007). Didaktik der Geometrie in der Sekundarstufe I, Hildesheim, Berlin : Franzbecker

Laborde, C. (1991). Zu einer Didaktik des Geometrie-Lernens unter Nutzung des Computers in Intelligente Tutorielle Systeme für das Lernen von Geometrie, Proceedings des Workshops am IDM, März 1991, Occasional Paper 124, Bielefeld, Germany: Universität Bielefeld, Institut für Didaktik der Mathematik.

Lagrange, J.-B. (2002). Etudier les mathématiques avec les calculatrices symboliques: quelles places pour les techniques ? In: In: Guin D. \& Trouche L. (eds.) Calculatrices symboliques, transformer un outil en un instrument du travail mathématique: un problème didactique (ch. 5, pp.151-185), Grenoble: La Pensée Sauvage Editions

Mariotti, A. (2001). Justifying and proving in the Cabri environment. International Journal of Computers for Mathematical Learning, 6(3), 257-281

Mithalal, J. (2010). Déconstruction instrumentale et déconstruction dimensionnelle dans le contexte de la géométrie dynamique tridimensionnelle. Thèse de l'Université Grenoble 1.

Moreno, J. (2006). Articulation des registres graphique et symbolique pour l'étude des équations différentielles avec Cabri-géomètre : analyse des difficultés des étudiants et rôle du logiciel. Thesis, Université Joseph Fourier, Grenoble, France.

Moreno Armella, L. (1999). Epistemologia ed Educazione matematica, La Matemetica e la sua Didattica, 1, 43-59

Netz, R. (1999). The shaping of deduction in greek mathematics Cambridge Univ. Press

Noss, R., \& Hoyles, C. (1996). Windows on Mathematical Meanings. Dordrecht, The Netherlands: Kluwer.

Parzysz, B. (1988). Knowing vs Seeing, Problems of representation of space geometry figures. Educational Studies in Mathematics, 19.1, 79-92

Restrepo, A. (2008). Genèse instrumentale du déplacement en géométrie dynamique chez des élèves de 6ème. Thèse Université Grenoble 1 
Smith, D., Harslem, E., Irby, C., \& Kimball, R. (1982). Designing the Star User Interface, Byte vol. 74, 242-282.

Schneidermann, B. (1983). Direct Manipulation: a step beyond programming languages, IEEE Computer, vol. 16, 57-69.

Strässer, R. (1991). Dessin et Figure Géométrie et Dessin technique à l'aide de l'ordinateur. (Juni 1991) Occasional paper $n^{\circ} 128$. Bielefeld, Germany: Universität Bielefeld, Institut für Didaktik der Mathematik.

Strässer, R. (1992). Didaktische Perspektiven auf Werkzeug-Software im Geometrie-Unterricht der Sekundarstufe I. Zentralblatt für Didaktik der Mathematik, 24(5), 197 - 201.

Vérillon, P. \& Rabardel, P. (1995). Cognition and artifacts. A contribution to the study of thought in relation to instrumented activity. European Journal of Psychology of Education 10(1), 77-101. 